\title{
Artificial Neural Networks and Fuzzy Neural Networks for Solving Civil Engineering Problems
}

\author{
Milos Knezevic $\mathbb{D}^{1},{ }^{1}$ Meri Cvetkovska $\left(\mathbb{D},{ }^{2}\right.$ Tomáš Hanák $\left(\mathbb{D},{ }^{3}\right.$ Luis Braganca $\left(\mathbb{D},{ }^{4}\right.$ \\ and Andrej Soltesz ${ }^{5}$ \\ ${ }^{1}$ University of Podgorica, Faculty of Civil Engineering, Podgorica, Montenegro \\ ${ }^{2}$ Ss. Cyril and Methodius University, Faculty of Civil Engineering, Skopje, Macedonia \\ ${ }^{3}$ Brno University of Technology, Faculty of Civil Engineering, Institute of Structural Economics and Management, \\ Brno, Czech Republic \\ ${ }^{4}$ Director of the Building Physics \& Construction Technology Laboratory, Civil Engineering Department University of Minho, \\ Guimaraes, Portugal \\ ${ }^{5}$ Slovak University of Technology in Bratislava, Department of Hydraulic Engineering, Bratislava, Slovakia \\ Correspondence should be addressed to Milos Knezevic; knezevicmilos@hotmail.com
}

Received 2 August 2018; Accepted 2 August 2018; Published 8 October 2018

Copyright ( 92018 Milos Knezevic et al. This is an open access article distributed under the Creative Commons Attribution License, which permits unrestricted use, distribution, and reproduction in any medium, provided the original work is properly cited.

Based on the live cycle engineering aspects, such as prediction, design, assessment, maintenance, and management of structures, and according to performance-based approach, civil engineering structures have to fulfill essential requirements for resilience, sustainability, and safety from possible risks, such as earthquakes, fires, floods, extreme winds, and explosions.

The analysis of the performance indicators, which are of great importance for the structural behavior and for the fulfillment of the above-mentioned requirements, is impossible without conducting complex mathematical calculations. Artificial neural networks and Fuzzy neural networks are typical examples of a modern interdisciplinary field which gives the basic knowledge principles that could be used for solving many different and complex engineering problems which could not be solved otherwise (using traditional modeling and statistical methods). Neural networks are capable of collecting, memorizing, analyzing, and processing a large number of data gained from some experiments or numerical analyses. Because of that, neural networks are often better calculation and prediction methods compared to some of the classical and traditional calculation methods. They are excellent in predicting data, and they can be used for creating prognostic models that could solve various engineering problems and tasks. A trained neural network serves as an analytical tool for qualified prognoses of the results, for any input data which have not been included in the learning process of the network. Their usage is reasonably simple and easy, yet correct and precise. These positive effects completely justify their application, as prognostic models, in engineering researches.

The objective of this special issue was to highlight the possibilities of using artificial neural networks and fuzzy neural networks as effective and powerful tools for solving engineering problems. From 12 submissions, 6 papers are published. Each paper was reviewed by at least two reviewers and revised according to review comments. The papers covered a wide range of topics, such as assessment of the real estate market value; estimation of costs and duration of construction works as well as maintenance costs; and prediction of natural disasters, such as wind and fire, and prediction of damages to property and the environment.

I. Marovic et al.'s paper presents an application of artificial neural networks (ANN) in the predicting process of wind speed and its implementation in early warning systems (EWS) as a decision support tool. The ANN prediction model was developed on the basis of the input data obtained by the local meteorological station. The prediction model was validated and evaluated by visual and common calculation approaches after which it was found out that it is applicable and gives very good wind speed predictions. The developed model is implemented in the 
EWS as a decision support for the improvement of the existing "procedure plan in a case of the emergency caused by stormy wind or hurricane, snow and occurrence of the ice on the University of Rijeka campus."

The application of artificial neural networks as well as econometric models is characterized by specific advantages and disadvantages. Nevertheless, neural networks have been imposed as a real alternative to econometric methods and as a powerful tool for assessment and forecasting, for example, in the field of evaluating real estate. It is specially emphasized that it is possible to find estimated values instead of exact values. The aim of J. Cetkovic et al.'s research was to construct a prognostic model of the real estate market value in the EU countries depending on the impact of macroeconomic indicators. Based on the available input data-macroeconomic variables that influence the determination of real estate prices, the authors sought to obtain fairly correct output data-prices forecast in the real estate markets of the observed countries.

Offer preparation has always been a specific part of a building process which has a significant impact on company business. Due to the fact that income greatly depends on offer's precision and the balance between planned costs, both direct and overheads, and wished profit, it is necessary to prepare a precise offer within the required time and available resources which are always insufficient. I. Peško et al.'s paper presents research on precision that can be achieved while using artificial intelligence for the estimation of cost and duration in construction projects. Both artificial neural networks (ANNs) and support vector machines (SVM) were analyzed and compared. Based on the investigation results, a conclusion was drawn that a greater accuracy level in the estimation of costs and duration of construction is achieved by using models that separately estimate the costs and the duration. The reason for this lies primarily in the different influence of input parameters on the estimation of costs in comparison with the estimation of duration of the project. By integrating them into a single model, a compromise in terms of the significance of input data is made, resulting in the lower precision of estimation when it comes to ANN models. SVM models feature a greater capacity of generalization, providing at the same time greater accuracy of estimation, both for the estimation of costs and duration of projects as well.

The same problem was treated by M. Juszczyk et al. Their research was on the applicability of ANN for the estimation of construction costs of sports fields. An applicability of multilayer perceptron networks was confirmed by the results of the initial training of a set of various artificial neural networks. Moreover, one network was tailored for mapping a relationship between the total cost of construction works and the selected cost predictors which are characteristic for sports fields. Its prediction quality and accuracy were assessed positively. The research results legitimate the proposed approach.

The maintenance planning within the urban road infrastructure management is a complex problem from both the management and the technoeconomic aspects. The focus of I. Marovic et al.'s research was on decision-making processes related to the planning phase during the management of urban road infrastructure projects. The goal of this research was to design and develop an ANN model in order to achieve a successful prediction of road deterioration as a tool for maintenance planning activities. Such a model was part of the proposed decision support concept for urban road infrastructure management and a decision support tool in planning activities. The input data were obtained from Circly 6.0 Pavement Design Software and used to determine the stress values. It was found that it is possible and desirable to apply such a model in the decision support concept in order to improve urban road infrastructure maintenance planning processes.

The fire resistance of civil engineering structures can be determined based on the estimated fire resistance of each construction element (columns, beams, slabs, walls, etc.). As fire resistance of structural elements directly affects the functionality and safety of the whole structure, the significance which new methods and computational tools have on enabling a quick, easy, and simple prognosis of the same, is quite clear. M. Lazarevska et al.'s paper considered the application of fuzzy neural networks by creating prognostic models for determining fire resistance of eccentrically loaded reinforced concrete columns. Using the concept of the fuzzy neural networks and the results of the performed numerical analyses (as input parameters), the prediction model for defining the fire resistance of eccentrically loaded RC columns incorporated in walls and exposed to standard fire from one side has been made. The numerical results were used as input data in order to create and train the fuzzy neural network so it can provide precise outputs for the fire resistance of eccentrically loaded RC columns for any other input data (RC columns with different dimensions of the cross-section, different thickness of the protective concrete layer, different percentage of reinforcement and for different loads).

These papers represent an exciting, insightful observation into the state of the art as well as emerging future topics in this important interdisciplinary field. We hope that this special issue would attract a major attention of the civil engineering's community.

We would like to express our appreciation to all the authors and reviewers who contributed to publishing this special issue.

\section{Conflicts of Interest}

As guest editors, we declare that we do not have a financial interest regarding the publication of this special issue.

Milos Knezevic

Meri Cvetkovska

Tomáš Hanák

Luis Braganca

Andrej Soltesz 


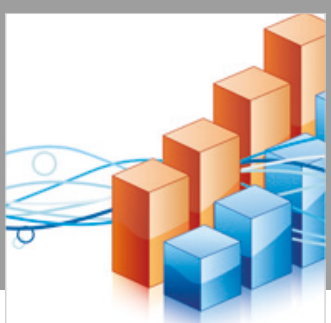

Advances in

Operations Research

\section{-n-m}
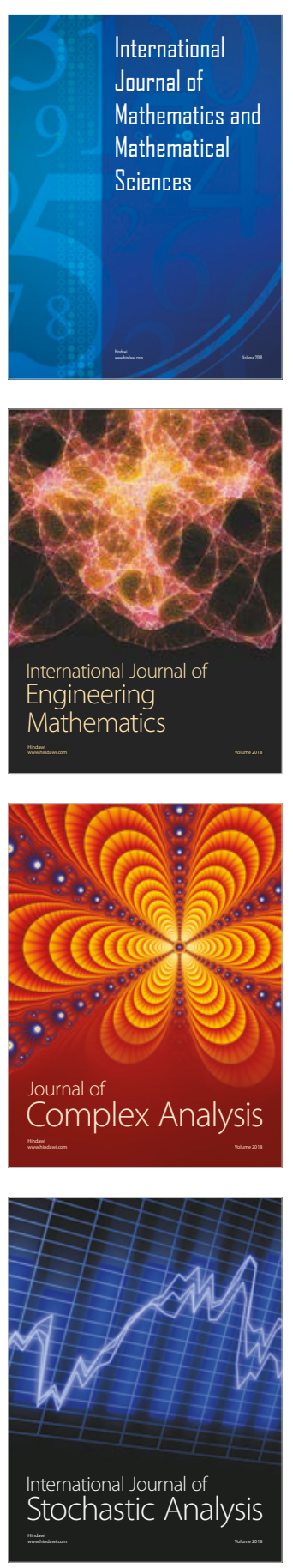
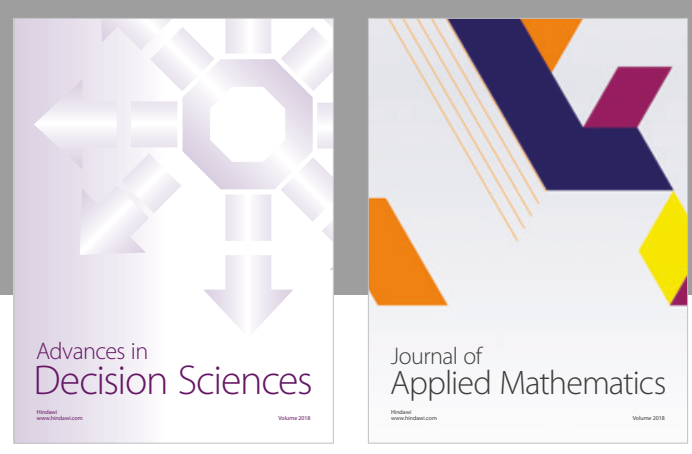

Journal of

Applied Mathematics
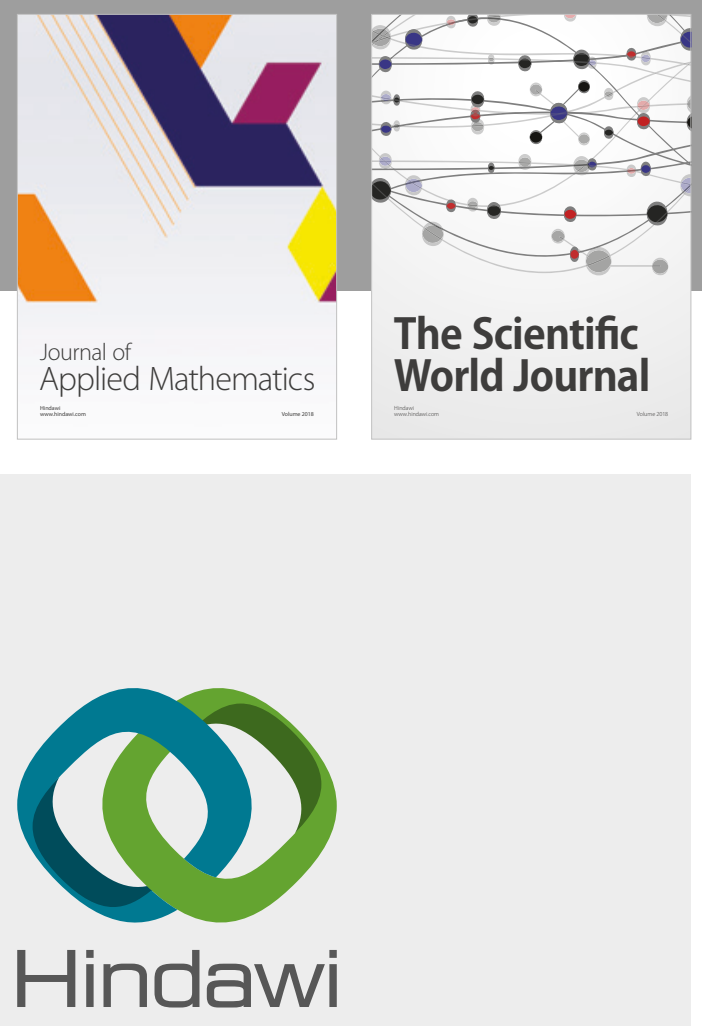

Submit your manuscripts at

www.hindawi.com

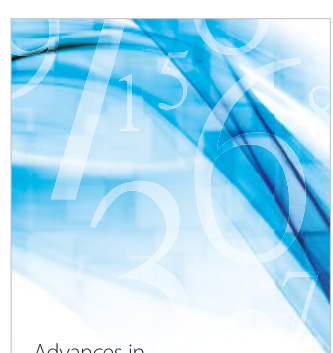

Advances in
Numerical Analysis
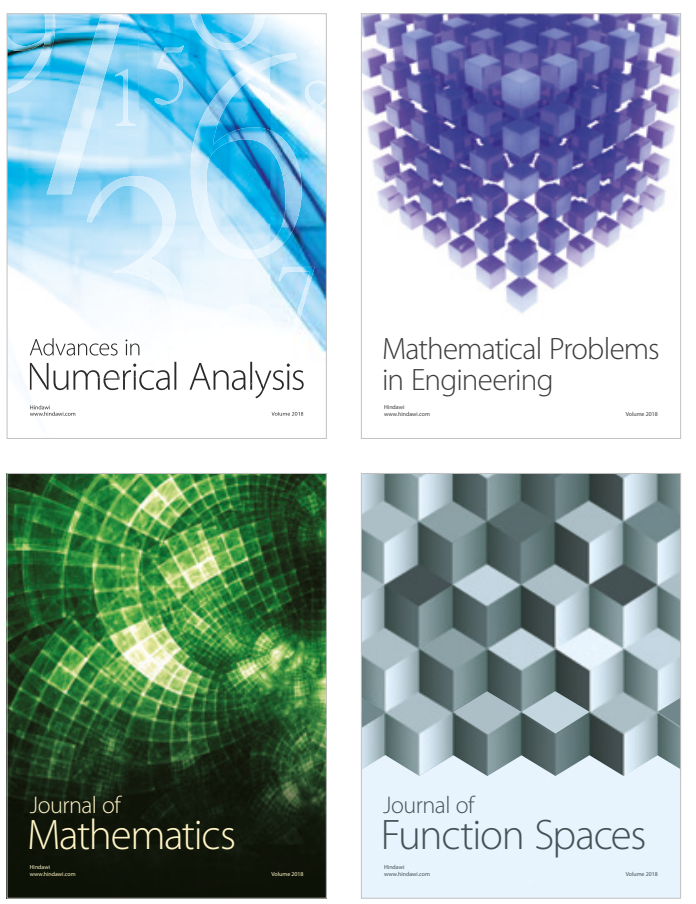

Mathematical Problems in Engineering

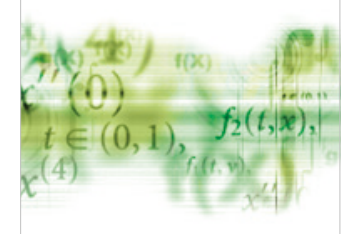

International Journal of

Differential Equations

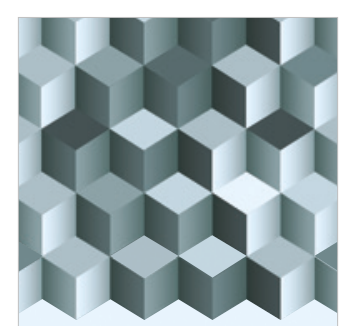

Journal of

Function Spaces
The Scientific

World Journal

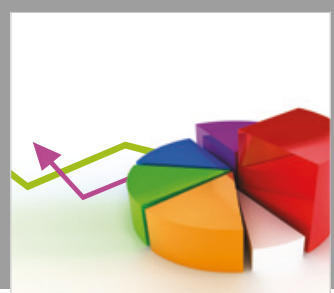

Journal of

Probability and Statistics
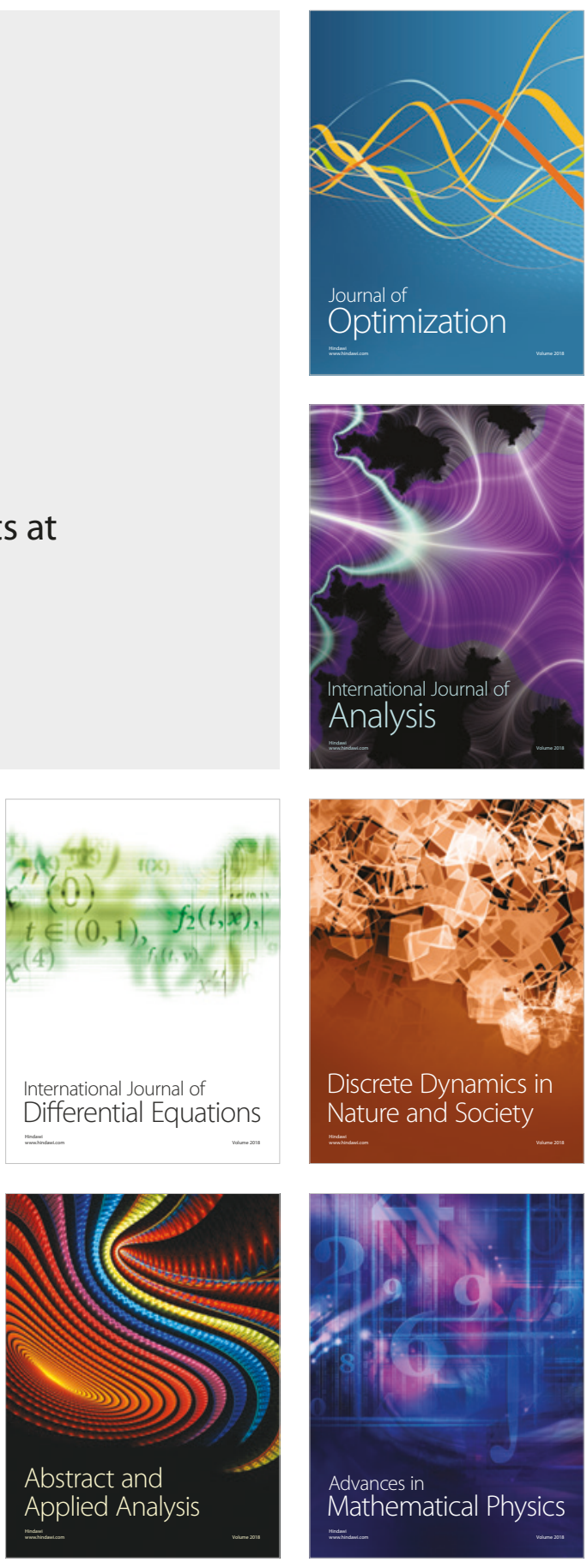